\title{
Constructing the Business Process of an Application System Based on Windows Workflow Foundation
}

\author{
Miao Cui, Jia Chen and Yu Jiang \\ School of Economics and Management, Dalian Maritime University, Dalian 16026, P.R \\ China Jackychen6662000@yaboo.com.cn chenjia_8008@sina.com \\ miaocui@newmail.dlmu.edu.cn
}

\begin{abstract}
The developing of the Application System always set the Business Process into the entire structure directly, each time the modification of the business process may make the program change largely. This structure adds the complexity to the system and hinders the flexibility of the system. Combining WWF and traditionally information system development theory to construct the Business Process can satisfy the need of the enterprise and make it easy to rebuild and update the Business Process.
\end{abstract}

Keywords: Business process modeling, Business transformation, Workflow analysis, Workflow model

\section{INTRODUCTION}

Today that is under the information-based tide, mostly business enterprise, especially the business enterprises of certain scale all carry on information-based construction. The developing of the Application System always set the Business Process into the entire structure directly, each time the modification of the business process may make the program change largely. This stiff structure increases the complexity of the system and baffles the flexibility of the system. The business enterprise which wants to exist under this exterior environment that full of competition and variety, have to meet the emergency with need, continuously adjusts itself, optimizes various business process of the business enterprise, and reconstructs the process. The integrated information management system should be an information collection, saving, handling, releasing and supporting the process reconstruction process, but the normal information management system is hard to do this.

The concept of the workflow comes into being under the construction of the modern information system. Workflow is a key technique which supports the business process reorganization and automation and can be supported and circulated by computer.

Please use the following format when citing this chapter

Cui, M., Chen. J., Jiang, Y.. 2007, in IFIP International Federation for Information Processing. Volume 254. Research and Practical Issues of Interprise Information Systems II Volume I. eds. I. Xu. Ijoa A.. Chaudhry S. (Boston: Springer), pp. 609-615. 


\section{WINDOWS WORKFLOW FOUNDATION}

\subsection{Workflow Model and Composition}

Microsoft Windows Workflow Foundation is a free, general and extensible framework, used for developing and performing application program that base on workflow. WWF provide a united platform for the products of Microsoft, customers and independent software developing company. It aims to provide a singular engine for workflow execution for all applications built on the Windows platform.

As a part of the upcoming Microsoft's next generation development Framework, WWF provides a workflow engine, a .NET trusteeship API (application program interface), runtime services, visible designer and debugger that integrate with Microsoft Visual Studio 2005, and can create and perform workflow that span client point and server point in the meantime, and can perform in all types .NET program.

\subsection{The Control of WWF to Process Reconstruction}

The most mature and most attraction side of workflow is the flexibility to set, perform and control process [1]. Figure 1 shows an examination and approve process model. In this process, carry on one class examination and approve to the application first, if pass to carry on the next operation, otherwise clue on do not pass. Figure 1
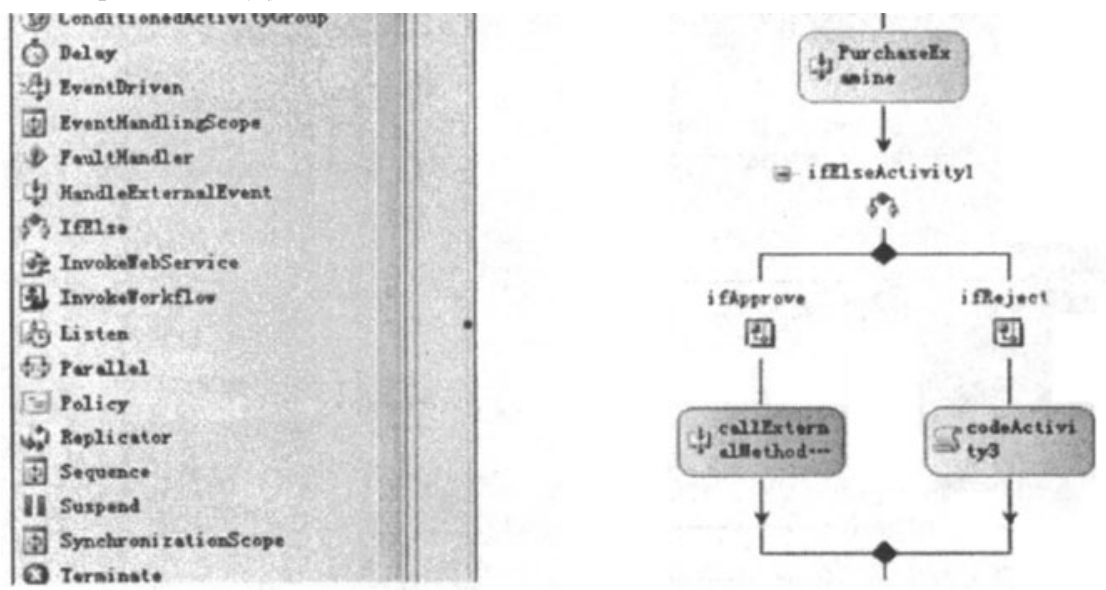

Figure 1. Old Examine Flow

shows the workflow model under WWF designer, as it shown, the left side of interface are some activity control, developer can add or delete them conveniently by dragging. 
Constructing the Business Process of an Application System Based on Windows Workflow

Foundation 611

For the need of management, one class examination and approve has already can't satisfy the requirement of the original examination, based on the one class examination and approve, carry on secondary class examination and approve. In the WWF designer it is very conveniently to make an improvement to the old process. Just adds an ifElse activity to the original process. The new model shown as the figure 2 , the activity callExternalMethod in figure 2 performs the same function as callExternalMethod in figure 1, the activity codeActivity 1 and codeActivity 3 in figure 2 also perform the same code as codeActivityl in figure 1 . CodeActivity 1 is the replication of codeActivity3. On the contrary, if the secondary class examine and approve dose not needed, can delete it from the model conveniently, and does not influence the normal work of other workflows.

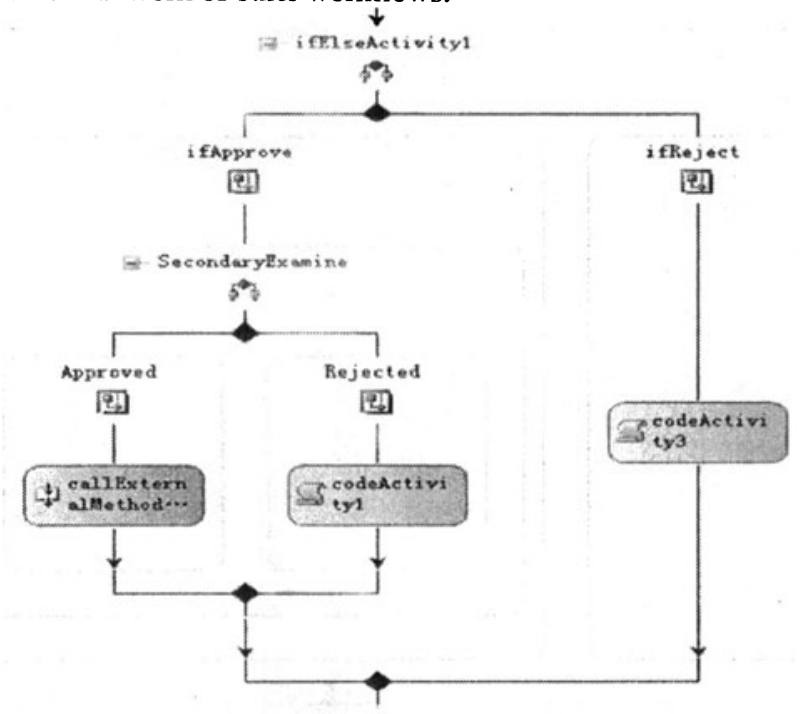

Figure 2. New Examine Flow

Therefore, the management information system which adopts WWF technique can consumedly raise the efficiency of the system develop, lower the development cost, promise the system better extensibility, flexibility.

\section{PROCESS MODELLING OF WORKFLOW}

\subsection{The Definition of UML Activity Diagram}

UML activity diagram is one of the graphics tools which is used to modelling for the dynamic behaviour of the system. UML activity diagram is substantively also a kind of flow chart, expresses the control flow from one activity to another, supports 
the expression of subsequent behaviour and conditional choice behaviour, and still supports the description of data flow. Especially suit the description of workflow.

UML activity diagram is a special kind of state machine, also a special kind of state graph. In a state graph, if most activities denote activity of the operation, and the transfer triggered by the activity, namely all or most events are performed by inner activities, is activity diagram [2]. Therefore, what activity diagram describes is the action of the object class which responds to the inner processing. It emphasizes the control flow from one activity to another. Usually, the activity diagram is on the supposition that there is no break off caused by the exterior events during the computer processing. UML activity diagram catch the result of the action (the work or activity that will be carried out) according to the variety of state. One activity will immediately get into the next activity after ending in an activity diagram.

\subsection{UML Modelling of Warehouse Management System}

The article will take warehouse management system for example, to explain the application of WWF in business process constructing. The system includes location management and in the location management module includes insert, delete, edit, and auto create location function.

Create location means based on the information of the selected warehouse (such as line, row, and layer) create the location circularly. If want to create location which have been created in the selected warehouse, should judge whether there are cargoes in the grid of the location first. If there are not cargoes, then can create, or can not create, the activity diagram shown as figure 3 :

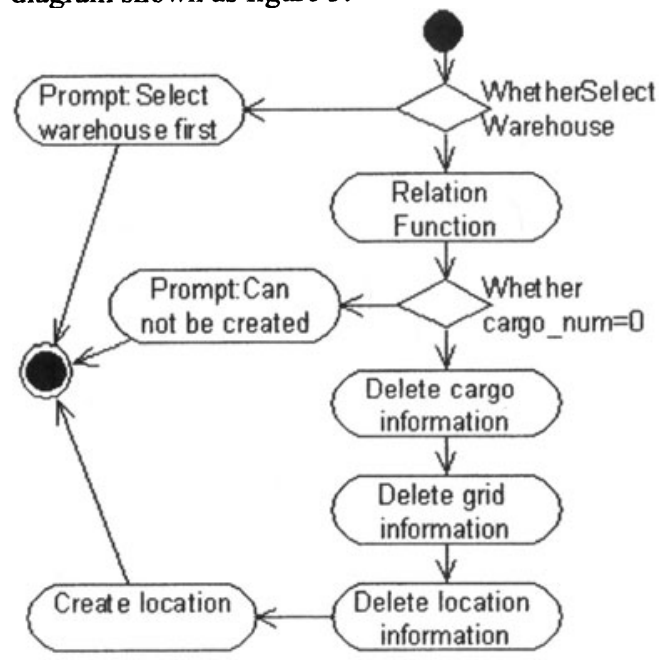

Figure 3. Activity Diagram of Creating Location 


\section{CARRY OUT WAREHOUSE MANAGEMENT SYSTEM}

\subsection{The Designing Thought of Process Management}

There are two models supported by WWF: Sequential workflow model comprising activities that execute in a predictable sequential path, and State machine model - a flow driven by events triggering state transitions [3].

There is no fixed model for designing workflow, take state machine model as main flow to control the state and take sequential workflow model as sub flow to carry out operation is not a bad way [4].

In the warehouse management system, include a lot of modules, each module perform different function and transfer many different sub modules, but what time is needed to transfer the sub modules is not sure beforehand. Thus adopt the state machine model is more suitable. In each state, the respond to the events can be carry out by sequential workflow model.

\subsection{The Designing and Realization of Process Management}

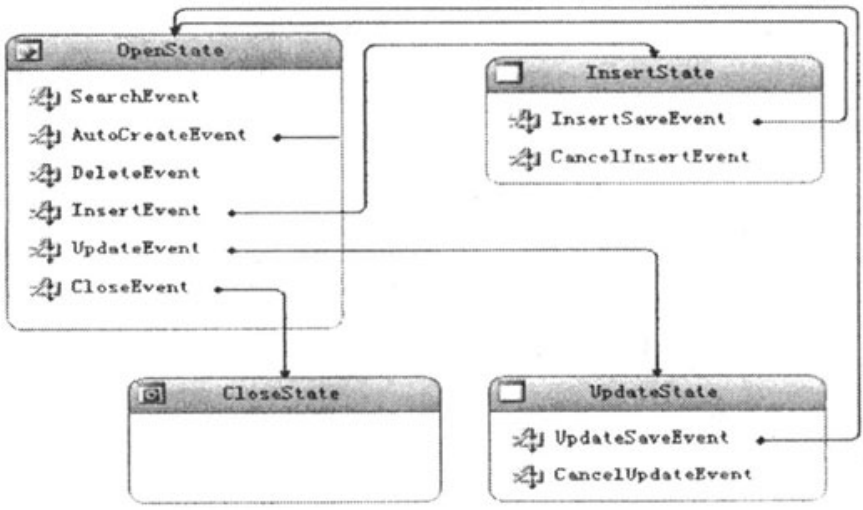

Figure 4. State Machine Model of Location Management

Location management module is a state, defined as initial state (OpenState). According to Figure 1, it includes search, insert, delete, edit and create function, and insert and edit function transfer insert and edit sub module which are defined as InsertState and UpdateState separately. InsertState and UpdateState are triggered by InsertEvent and UpdateEvent in the initial state. The events InsertSaveEvent and UpdateSaveEvent in the sub module perform save operation that is send the data into the database, and return to the initial state after operating successfully. The state machine model described shown as Figure 4. 
The event AutoCreateEvent in the initial state is used for performing create location function. In the article 3.2 , the activity diagram of create location has been given, still take it for example, the corresponding sequential workflow to Figure 4 shown as Figure 5:

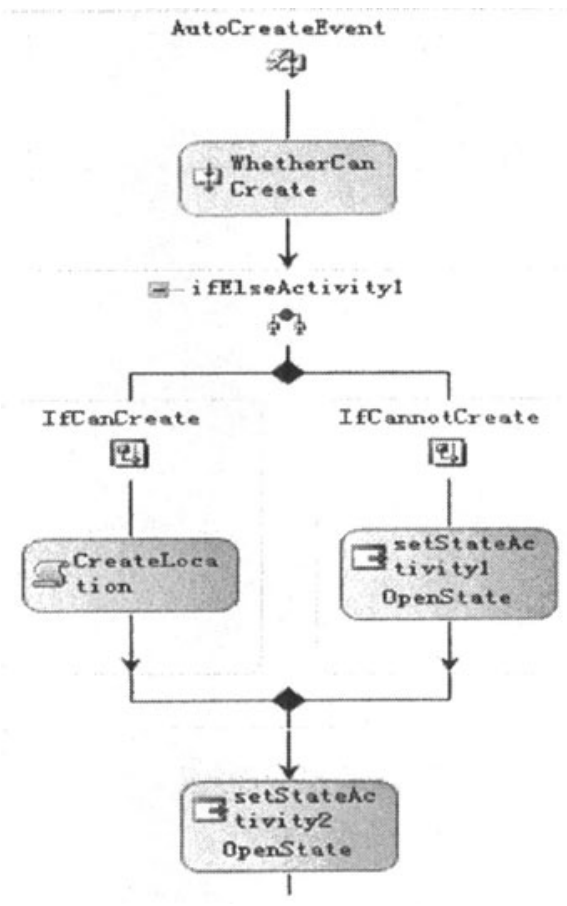

Figure 5. Sequential Workflow of Create Location

Event WhetherCanCreate judge whether can create location by implement the event FunRelationLocation of the interface IAutoCreate, if there are no cargoes in the grid, then implement the left side flow of ifElse, and circularly create location by implement code Createlocation which include delete the information of the cargo, grid, and location, if there are still cargoes in the grid, then implement the right side flow of ifElse and the flow return to the initial state.

\section{CONCLUSIONS}

The article makes use of WWF framework which is released by Microsoft lately to construct warehouse management system based on .NET platform, constructs UML model of the location management module, and introduces a visible designing method based on WWF model. Combining traditionally developing technology of system and 
Constructing the Business Process of an Application System Based on Windows Workflow Foundation 615 workflow technology make the manager supervise and optimize the flow exactly and conveniently, and make the business enterprise acquire larger income.

\section{REFERENCES}

1. F. Yushun, Workflow Management Foundation (Qinghua University Press: Beijing, 2001).

2. J. Arlow and I. Neustadt, UML2 and the Unified Process (China Machine Press: Beijing, 2006).

3. V. Yen, A node-centric analysis of metagraphs and its applications to workflow models, Enterprise Information Systems. Volume 1, Number 1, pp.139-159, (2007).

4. P. Andrew and J. Conard, Presenting Windows Workflow Foundation, Sams Publishing (2006). http://msdn.microsoft.com/workflow (Accessed October 1, 2006). 